\title{
The Royal College of Ophthalmologists' National Ophthalmology Database study of cataract surgery: report 4 , equity of access to cataract surgery
}

\author{
Robert L. Johnston ${ }^{1,2} \cdot$ Alexander C. Day $\mathbb{1}^{3} \cdot$ Paul H. J. Donachie ${ }^{1,2} \cdot$ John M. Sparrow ${ }^{1,4}$ \\ Received: 29 November 2017 / Revised: 24 February 2019 / Accepted: 25 May 2019 / Published online: 29 July 2019 \\ (c) The Author(s), under exclusive licence to The Royal College of Ophthalmologists 2019
}

\begin{abstract}
Objective To determine whether socioeconomic status influenced the presenting visual acuity prior to first eye cataract surgery in the English National Health Service. Retrospective case series from The Royal College of Ophthalmologists' National Ophthalmology Database Audit. In total 154,223 patients undergoing first eye cataract surgery at 68 centres in England performed between 1st September 2015 and 31st August 2017.

Main outcome measure Social deprivation status and pre-operative visual acuity (VA) between centres for patients undergoing first eye cataract surgery in England.

Results The median social deprivation varied between centres and ranged from decile 2 (2nd most deprived decile) to decile 9 (2nd least deprived decile). The pre-operative VA was reported for 143,401 (93.0\%) eyes. The median pre-operative VA was $0.50 \operatorname{LogMAR}(6 / 19)$, and $27.7 \%$ eyes had a preoperative VA of $0.30 \operatorname{LogMAR}$ units $(6 / 12)$ or better. The median preoperative VA for each centre ranged from 0.30 to $0.60 \operatorname{LogMAR}(6 / 12$ to $6 / 24)$. The median pre-operative VA was mostly stable across deciles of social deprivation (0.60 LogMAR for decile 1 and $0.50 \operatorname{LogMAR}$ for all other deciles), and some evidence was found linking greater deprivation to worse pre-operative VA and to lower levels of access.

Conclusions We found no strong evidence of inequality for gaining access to first eye cataract surgery in this National Ophthalmology Database analysis, however there was a possible trend towards fewer people in the more deprived deciles accessing surgery, and that some of these are presenting with quite marked levels of visual impairment.
\end{abstract}

\section{Introduction}

The key principles of the NHS constitution include providing a comprehensive service, available to all, based on clinical need and not the ability to pay [1]. The constitution makes clear that "it has a wider social duty to promote equality

John M. Sparrow

john.sparrow@doctors.org.uk

1 The Royal College of Ophthalmologists' National Ophthalmology Audit, 18 Stephenson Way, London NW1 2HD, UK

2 Gloucestershire Hospitals NHS Foundation Trust, Cheltenham GL53 7AN, UK

3 The NIHR Biomedical Research Centre at Moorfields Eye Hospital NHS Foundation Trust and UCL Institute of Ophthalmology, London, UK

4 Bristol Eye Hospital, Lower Maudlin Street, Bristol BS1 2LX, UK through the services it provides and to pay particular attention to groups or sections of society where improvements in health and life expectancy are not keeping pace with the rest of the population." There has been relatively little work to date in the UK to determine whether these constitutional commitments are being upheld in relation to cataract surgery, with contrasting findings reported [2-5].

The aims of this study are to investigate if socioeconomic status is associated with pre-operative visual acuity (VA) in patients undergoing first eye cataract surgery in the English NHS and to consider differences in this regard between individual NHS Trusts.

\section{Methods}

The Royal College of Ophthalmologist's National Ophthalmology Database Audit (RCOphth NOD) conducts the annual National Cataract Audit (NOA) and receives 
pseudoanonymised data from participating NHS Trusts in England and Cymru. The data is recorded on electronic medical record systems (EMR) or in-house databases and extracted in the autumn of each year. The audit year runs from 1st September to 31st August and the audit brief is to report on NHS funded cataract surgery, including that performed in independent treatment centres.

The NOA is an audit of cataract surgery using phacoemulsification to treat patients aged 18 years or older, where the primary intention was cataract surgery and not combined 'cataract + other' surgery, unless the 'other' surgery formed part of the cataract operation (e.g. an operative manoeuvre to increase the size of the pupil).

The analysis presented in this manuscript concerns audit eligible cataract operations performed in England in the first two prospective audit years (01/09/2015-31/08/ 2017), using data recorded on the Medisoft EMR system (Medisoft Ophthalmology, Medisoft Limited, Leeds, UK, www.medisoft.co.uk). The operations recorded on other data collection systems are excluded as none of the centres using these other systems supplied social deprivation data during these two prospective audit years. Operations from centres in Cymru are excluded as the social deprivation indices are different in Cymru and there were only 2 participating centres from Cymru in the first two prospective audit years.

The Index of Multiple Deprivation (IMD) is the official measure of relative deprivation for small areas (neighbourhoods) in England. It ranks every small area in England from 1 (most deprived area) to 32,844 (least deprived area) [6]. From these ranks national deciles of social deprivation are created. The IMD score, rank and deciles were automatically created during data extraction from the patient's postcode, which itself was not transferred to the RCOphth NOD.

Pre-operative VA was defined as the better measurement of corrected distance VA or uncorrected distance VA that is closest to the date of surgery, including the day of surgery and within 4 months prior to surgery. Pin hole VA measurements were not used. This study restricted the analysis to the patient's first eye cataract operation reported to the NOA with a calculable IMD score, rank and decile.

The lead clinician and Caldicott Guardian (responsible nominee for data protection) at each NHS Trust gave written approval for anonymised data extraction. Anonymized database analyses of this type do not require ethical permission as they are viewed as audit or service evaluation (see http://www. hra.nhs.uk/research-community/before-you-apply/determinew hether-your-study-is-research/). This study was conducted in accordance with the declaration of Helsinki, and the UK's Data Protection Act.

\section{Results}

\section{Eligibility and sample}

For the first and second prospective audit years, the RCOphth NOD received 162,502 eligible first eye cataract operations recorded on the Medisoft EMR from 70 participating centres in England. Of these, 8279 (5.1\%) operations were excluded from this analysis, 5869 (3.6\%) operations from 2 centres that withdrew permission to publish their data after identifying data quality issues, and $2410(1.5 \%)$ operations with missing social deprivation data.

Following exclusions, a single eligible first eye cataract operation was available from 154,223 patients from 68 participating centres, where the median number of operations per centre was 3905 (range; 39-9245).

The median (range) age was 76.3 years $(18.1-113.9$ years), $65,798(42.7 \%)$ patients were male, $88,115(57.1 \%)$ were female and the gender was not stated for $310(0.2 \%)$ patients.

\section{Social deprivation}

For the sample of 154,223 patients, the percentage of patients from each national decile of social deprivation was $9.4 \%, 8.8 \%, 9.1 \%, 9.7 \%, 9.7 \%, 10.2 \%, 10.3 \%, 10.6 \%$, $10.7 \%$, and $11.5 \%$ for deciles $1-10$ respectively, suggesting higher levels of access among less deprived individuals.

All bar 6 centres performed cataract surgery in eyes from patients from all national deciles of social deprivation, 4 centres performed no cataract operations in patients in the most deprived decile (decile 1) and 2 centres no cataract operations in patients in the least deprived decile (decile 10). For centres, the median social deprivation decile of their patients ranged from the most deprived centre, decile 2 to the least, decile 9, Fig. 1.

\section{Pre-operative visual acuity}

A presenting VA was recorded for 143,783 (93.2\%) eyes and missing for $10,440(6.8 \%)$ eyes. The percentage of eyes with a missing pre-operative VA varied between participating centres, where $45(66.2 \%)$ centres had $<10 \%$ of eyes with a missing pre-operative VA, and $5(7.4 \%)$ centres $>40 \%$, Fig. 2. The proportion of eyes with a missing preoperative VA was associated with social deprivation with lower proportions with a missing pre-operative VA for the least deprived deciles; $7.2 \%, 7.6 \%, 7.4 \%, 7.1 \%, 6.6 \%$, $6.5 \%, 6.5 \%, 7.0 \%, 6.4 \%$, and $5.8 \%$ for deciles $1-10$ respectively, $p<0.001$. Of the 143,783 eyes with a recorded pre-operative VA, $382(0.3 \%)$ operations from 5 centres are 


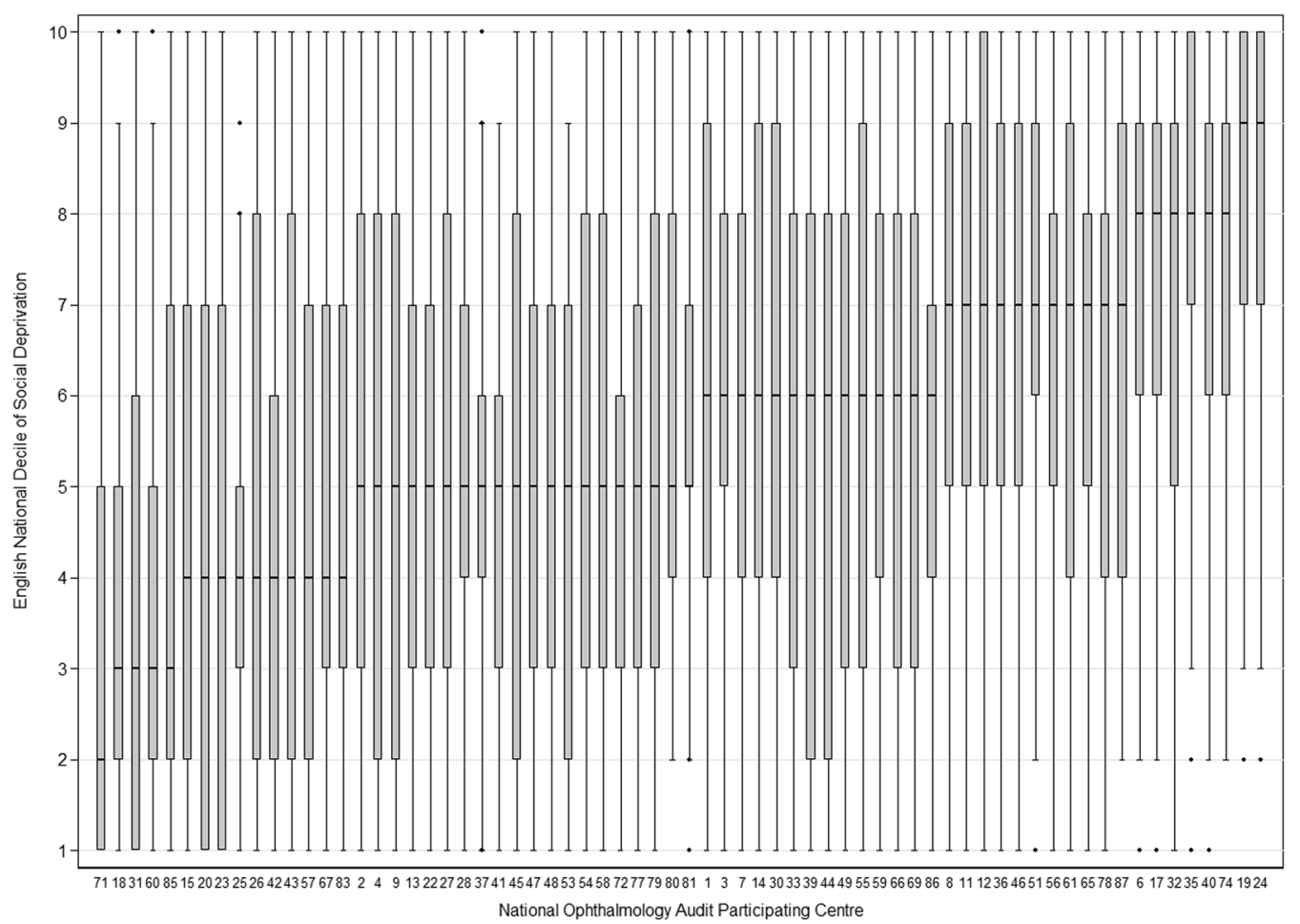

Fig. 1 Box and whisker plots of national deciles of social deprivation for England by participating centre and ordered by median decile. The national deciles of social deprivation indicate the most deprived $10 \%$ (decile 1) up to the least deprived 10\% (decile 10) of England. The 'box' displays the inter-quartile range (IQR) with the median in the middle, the whiskers (extending lines) stop at $1.5 \times \mathrm{IQR}$ and the markers indicate extreme values outside of $1.5 \times \mathrm{IQR}$. $N=154,223$ patients undergoing first eye cataract surgery in 68 participating centres

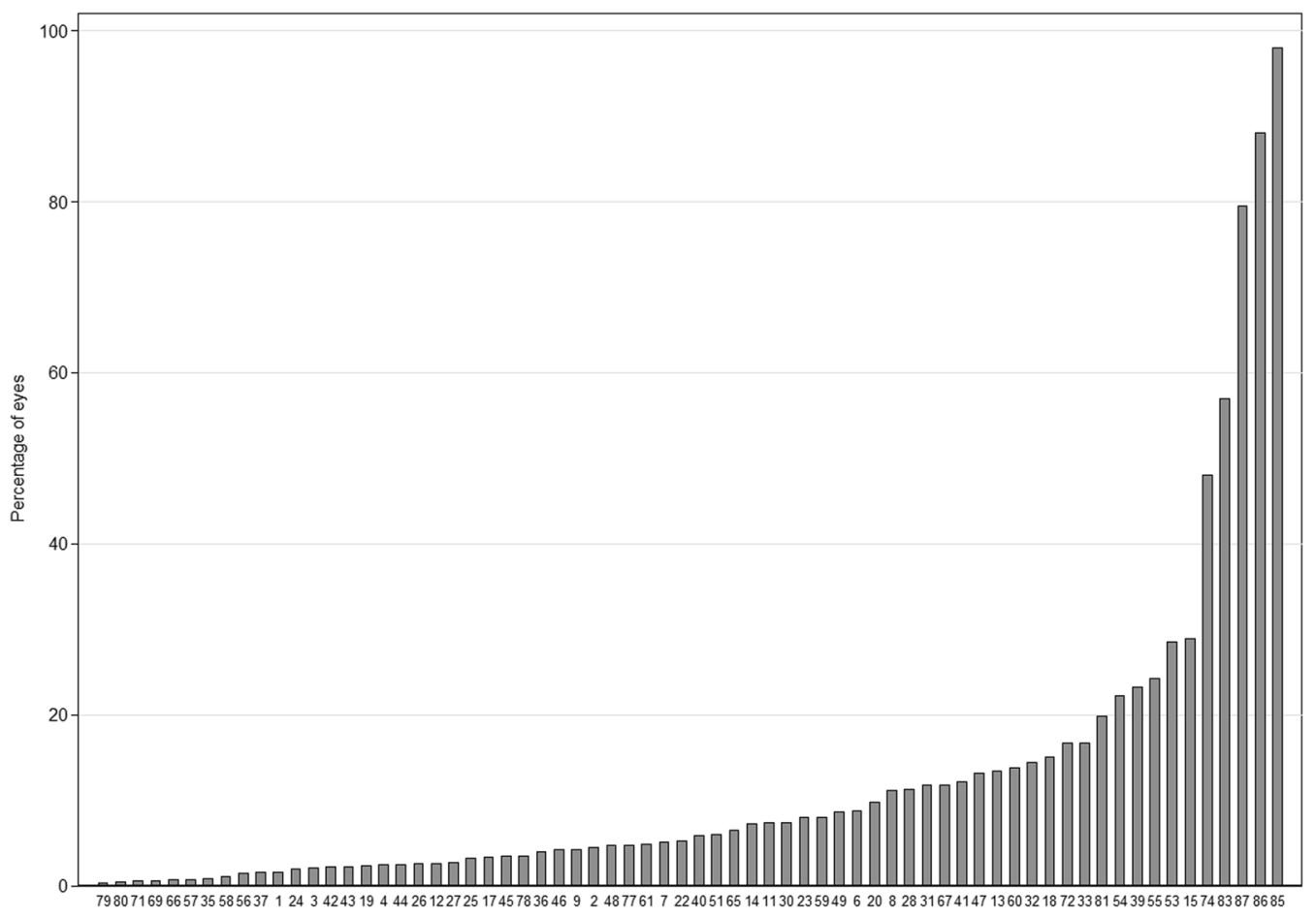

National Ophthalmology Audit Participating Centre

Fig. 2 Bar chart of the percentage of eyes with a missing pre-operative visual acuity by participating centre and ordered by the percentage with missing data. $N=154,223$ patients undergoing first eye cataract surgery in 68 participating centres 
not included in the VA results as these centres had $>40 \%$ of operations with a missing VA.

From 143,401 operations performed in 63 centres, the VA measurement was corrected distance VA in 107,551 $(75.0 \%)$ eyes, uncorrected distance VA in $33,647(23.5 \%)$ eyes and in $2203(1.5 \%)$ eyes the corrected distance VA was the same as the uncorrected distance VA.

The median pre-operative VA was 0.50 LogMAR (range; -0.30 - NPL) (Snellen equivalent 6/19), where $5804(4.0 \%)$ eyes were 'count fingers', 3327 (2.3\%) eyes were 'hand movements', $831(0.6 \%)$ eyes were 'perception of light' and $61 \quad(<0.1 \%)$ eyes 'no perception of light' (NPL).

The preoperative VA was 0.30 LogMAR units (6/12) or better for 39,753 $(27.7 \%)$ eyes, 0.60 LogMAR units $(6 / 24)$ or better for $97,021(67.7 \%)$ eyes and 1.0 LogMAR units (6/ $60)$ or better for $126,585(88.3 \%)$ eyes.

The median pre-operative VA for each centre ranged from 0.30 to $0.60 \operatorname{LogMAR}(6 / 12$ to $6 / 24)$ and for 48 centres the median pre-operative VA was 0.50 LogMAR, Fig. 3.

The median pre-operative VA was stable across all but one of the deciles of social deprivation, where the median pre-operative VA was 0.60 LogMAR for the most deprived decile (decile 1) and 0.50 LogMAR for all other deciles, Fig. 4. By grouping pre-operative VA into $<0.30$ LogMAR, $0.30-<0.60$ LogMAR, $0.60-<1.00$ LogMAR and $\geq 1.00$ LogMAR a different pattern is present. Generally, a higher proportion of patients from least deprived deciles had a better pre-operative VA, this difference was most marked for eyes with the worst visual acuity, where $23.6 \%$ of those in the most deprived decile had a pre-operative VA of $\geq 1.00 \operatorname{LogMAR}$ vs. $12.5 \%$ of those in the least deprived decile, Table 1.

\section{Discussion}

This study on a very large sample of patients accessing NHS cataract surgery at 68 centres in England includes data from approximately $50 \%$ of traditional centres in England performing NHS funded cataract surgery during the time period. Overall, whilst it is heartening to report that the NHS constitution's ambition to provide equity of access regardless of socioeconomic status appears a reality overall for the majority of people undergoing cataract surgery, we found a possible trend that fewer people in the more deprived deciles were accessing surgery, and were presenting for their first cataract operation with worse preoperative VA, for these two reported NOD audit years.

In this cohort the proportions of patients undergoing first eye surgery varied within deprivation deciles between $8.8 \%$ and $11.5 \%$. Although there was the suggestion of a trend implying that fewer people in the more deprived deciles were accessing surgery, this observation should be interpreted with caution as it may have resulted from a biased cohort where there were simply fewer participating centres in localities with high deprivation. In the present study, the median presenting VA before cataract surgery was identical at $0.50 \operatorname{LogMAR}$ for all bar the most deprived decile of social deprivation status. The socioeconomic case mix of patients presenting to different hospitals varies between hospitals, which mirrors the geographically varied social deprivation status across England, with only 6 of 68 centres not performing operations on patients from all deciles of social deprivation. We found evidence that worse pre-operative VA was associated with greater deprivation, with for example, almost a quarter of patients in the most deprived $10 \%$ (decile 1) having a presenting visual acuity of $\geq 1.00$ LogMAR compared to 1 in 8 patients from the least deprived $10 \%$ (decile 10). It may be that local factors in certain more deprived areas which limit access to surgery on the basis of VA are disproportionately affecting the most deprived individuals. It should however be borne in mind that these results are affected by the varied percentages of missing preoperative VA between centres, which ranged from $0.3 \%$ to $28.9 \%$ for the 63 centres with VA measurement data reported, and a 5 further centres not included due to $>40 \%$ with a missing VA. This will partly be due to differing modes of usage of the EMR systems between centres (e.g. recent adopters may initially use the EMR only in the operating theatre) and further research is required.

In contrast to our findings, Keenan et al. previously reported that the rates of cataract surgery by local authority area were positively correlated with the index of multiple deprivation score [2] suggesting that high rates of surgery were available in areas with high levels of social deprivation [2]. Work by Whillans and Nazroo found no evidence of discrimination of access to cataract surgery on the grounds of individuals' material or social position over an 8 year observation study (2002-2010) [4]. However a recent national survey of 151 local cataract surgery commissioners found that almost half had policies restricting access to cataract surgery with many denying treatment to those expected to benefit [3]. A third made no allowance for second eye cataract surgery [3]. They also reported that 92\% local commissioners used criteria that did not follow national guidance or research evidence [3]. A recent analysis of Hospital Episode Statistics in England found fewer cataract surgeries were performed in areas with low socioeconomic status, and also reported there was a $5 \%$ decline in the rate of cataract surgeries in the first year (fiscal year 2011) of a major efficiency saving programme in the NHS [5]. 


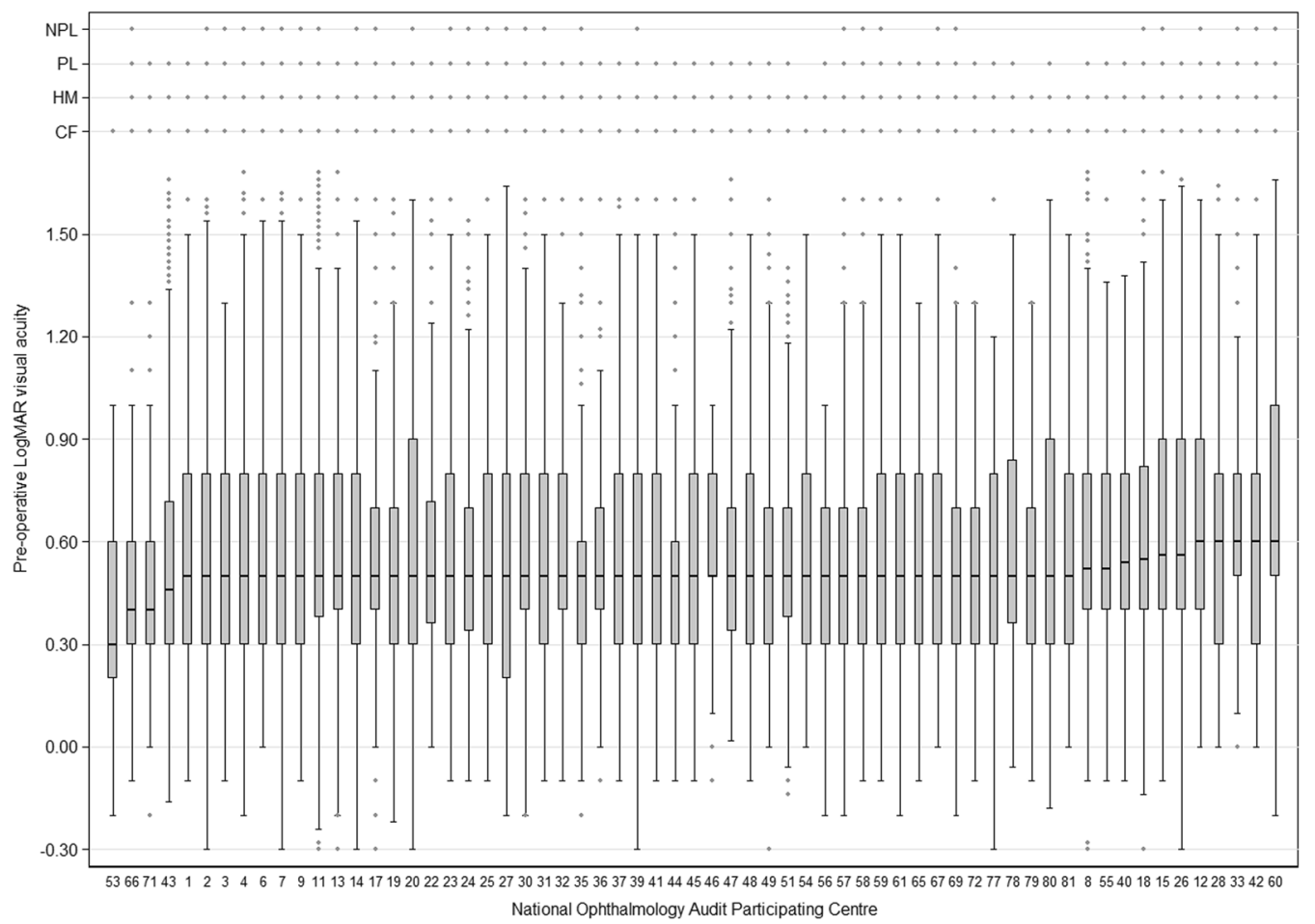

Fig. 3 Box and whisker plots of LogMAR pre-operative visual acuity by participating centre and ordered by median LogMAR visual acuity. The 'box' displays the inter-quartile range (IQR) with the median in the middle, the whiskers (extending lines) stop at $1.5 \mathrm{x}$ IQR and the markers indicate extreme values outside of 1.5 x IQR. $N=143,401$ patients undergoing first eye cataract surgery in 63 participating centres

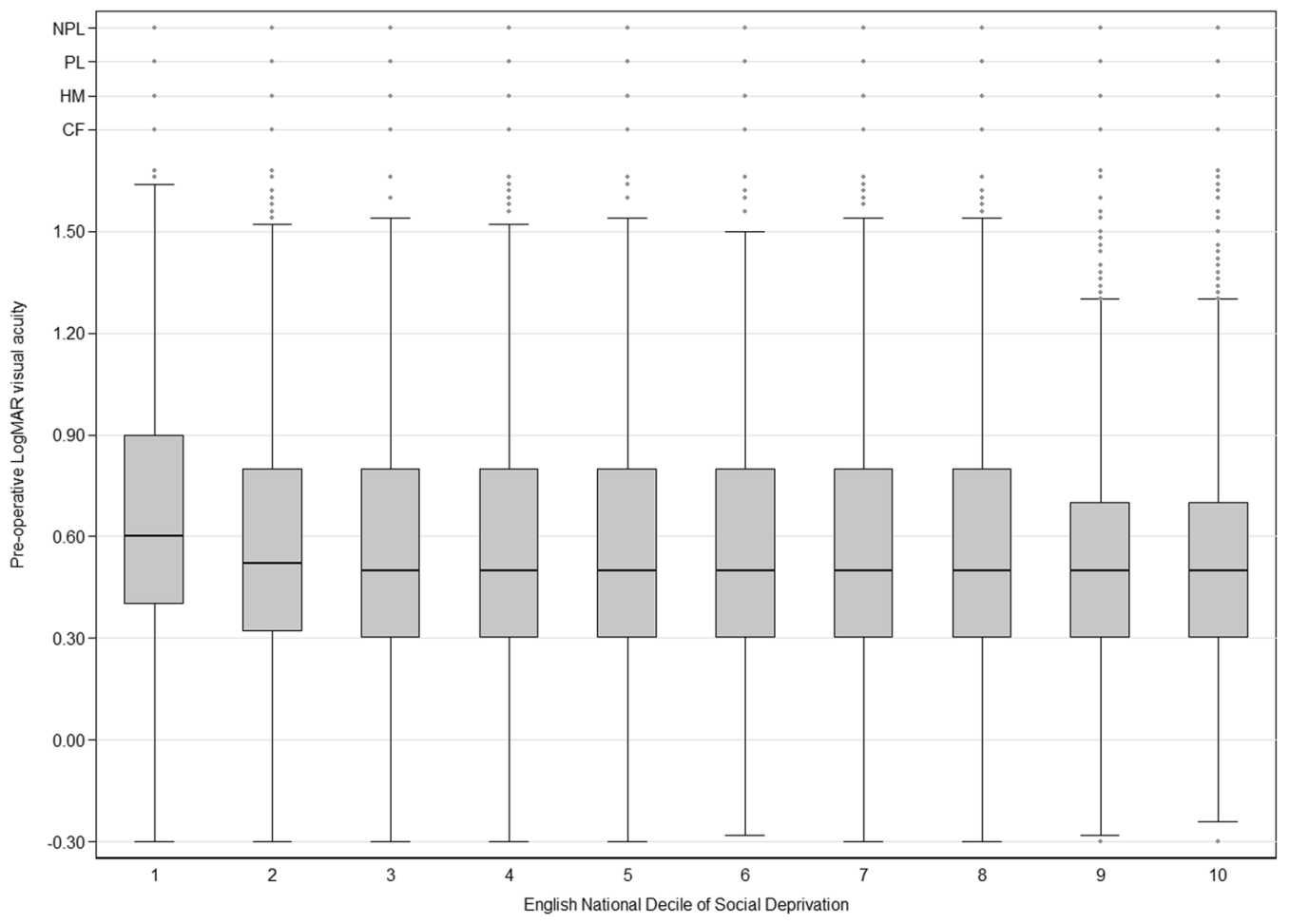

Fig. 4 Box and whisker plot of LogMAR pre-operative visual acuity by national deciles of social deprivation for England. The national deciles of social deprivation indicate the most deprived $10 \%$ (decile 1) up to the least deprived $10 \%$ (decile 10) of England. The 'box' displays the inter-quartile range (IQR) with the median in the middle, the whiskers (extending lines) stop at $1.5 \times \mathrm{IQR}$ and the markers indicate extreme values outside of 1.5 x IQR. $N=143,401$ patients undergoing first eye cataract surgery in 63 participating centres 
Table 1 Pre-operative VA for deciles of social deprivation

\begin{tabular}{lrrrrr}
\hline & \multicolumn{5}{c}{$\begin{array}{c}\text { Percentage with a pre-operative } \\
\text { VA of (logMAR) }\end{array}$} \\
\cline { 3 - 6 } $\begin{array}{l}\text { Decile of social } \\
\text { deprivation }\end{array}$ & $N$ & $<0.30$ & $0.30-$ & $0.60-$ & $\geq 1.00$ \\
\hline 1 & 13,372 & 9.8 & 39.0 & 27.6 & 23.6 \\
2 & 12,543 & 10.4 & 40.3 & 27.5 & 21.8 \\
3 & 12,908 & 10.0 & 43.4 & 27.1 & 19.5 \\
4 & 13,914 & 10.4 & 42.8 & 27.9 & 18.9 \\
5 & 13,874 & 11.3 & 44.1 & 27.5 & 17.1 \\
6 & 14,673 & 10.1 & 46.6 & 26.8 & 16.4 \\
7 & 14,842 & 11.2 & 46.8 & 26.3 & 15.6 \\
8 & 15,146 & 11.6 & 47.3 & 26.2 & 15.0 \\
9 & 15,457 & 11.2 & 49.1 & 25.9 & 13.7 \\
10 & 16,672 & 11.2 & 50.5 & 25.9 & 12.5 \\
Overall & $\mathbf{1 4 3 , 4 0 1}$ & $\mathbf{1 0 . 8}$ & $\mathbf{4 2 . 3}$ & $\mathbf{2 6 . 8}$ & $\mathbf{1 7 . 2}$ \\
\hline
\end{tabular}

The national deciles of social deprivation indicate the most deprived $10 \%$ (decile 1) up to the least deprived 10\% (decile 10) of England

Evidence from other healthcare systems around the world indicate that there are sometimes marked differences in access to cataract surgery according to socioeconomic status. The UK experience of apparent near equity of access to cataract surgery regardless of socioeconomic status is mirrored in Sweden and Australia. In the Blue Mountains Eye Study from Australia of 3654 participants, there was no significant association between incident cataract surgery and major socioeconomic categories [7]. Data from Sweden in 2016 found longer waiting times were associated with good VA, female sex, older age, retired, being born outside the Nordic countries and having lower income or education [8]. The authors concluded that these finding although statistically significant were on average relatively small and unlikely to be clinically important [8]. By contrast in the USA, an analysis of the Hispanic population in the Proyecto VER population study, found high rates of visually symptomatic cataract and concluded that language and financial barriers impeded access to cataract surgery [9]. More recent data from a Canadian analysis of 1350 eyes undergoing cataract surgery by a single surgeon reported patients of lower socioeconomic status had more advanced cataracts at the time of surgery [10].

The limitations of this study included that not all contributing centres to the National Audit supplied social deprivation data which restricted the analysis to the 68 $(81.0 \%)$ centres all using the same EMR system who supplied social deprivation data. The sample constitutes patients undergoing cataract surgery in $\sim 50 \%$ of traditional NHS funded cataract surgery centres in England during the time period. Data was not available on any patient who had been denied cataract surgery or the time period of visual loss or sight impairment prior to cataract surgery. Another limitation is that this analysis does not include data on cataract surgeries performed in the private sector and higher rates of cataract surgery performed in the private sector would be expected in areas of lower deprivation. Although this is not a sufficient proportion for definitive national conclusions, the findings are cautiously encouraging and with future expansion of the coverage of the national audit a more comprehensive picture is expected to emerge.

In summary, we found no strong evidence of inequality for gaining access to cataract surgery in this National Ophthalmology Database analysis of 154,223 first eye operations performed in 68 NHS centres. However there was a possible trend towards fewer people in the more deprived deciles accessing surgery, and that some of these more deprived individuals are presenting with quite marked levels of visual impairment at least for these two prospective NOD audit years. Further research is required to gain a more comprehensive UK wide national picture.

\section{Summary}

\section{What was known before}

- Rates of cataract surgery by area are positively correlated with the index of multiple deprivation score.

\section{What this study adds}

- There was a possible trend towards fewer people in the more deprived deciles accessing surgery, and that some of these more deprived individuals are presenting with quite marked levels of visual impairment.

- There is some variation in pre-operative visual acuity across deprivation deciles and between centres, which may indicate variable local resource issues affecting access to surgery.

Acknowledgements It is with deep regret that we note the death of our friend and colleague Robert Johnston, who sadly died in September 2016. Without his inspirational vision, determination and career long commitment to quality improvement in ophthalmology this work would not have been possible. We would like to acknowledge the support and guidance we have received from the National Audit Steering Committee which includes professional members, ophthalmologists and optometrists, and patient and public representatives with individual lay members as well as patient support groups being represented. We acknowledge the support of the hospitals that participated in the National Ophthalmology Database Audit and thank our medical and non-medical colleagues for the considerable time and 
effort devoted to data collection. The 68 participating centres included in this study are listed in alphabetic order below. Aintree University Hospital NHS Foundation Trust; Barking, Havering and Redbridge University Hospitals NHS Trust; Barts Health NHS Trust; Blackpool Teaching Hospitals NHS Foundation Trust; Bradford Teaching Hospitals NHS Foundation Trust; Calderdale and Huddersfield NHS Foundation Trust; Manchester University NHS Foundation Trust; Chesterfield Royal Hospital NHS Foundation Trust; County Durham and Darlington NHS Foundation Trust; East Lancashire Hospitals NHS Trust; East Sussex Healthcare NHS Trust; Epsom and St Helier University Hospitals NHS Trust; Frimley Health NHS Foundation Trust; Gloucestershire Hospitals NHS Foundation Trust; Great Western Hospitals NHS Foundation Trust; Hampshire Hospitals NHS Foundation Trust; Harrogate and District NHS Foundation Trust; Imperial College Healthcare NHS Trust; Isle of Wight NHS Trust; James Paget University Hospitals NHS Foundation Trust; King's College Hospital NHS Foundation Trust; Kingston Hospital NHS Foundation Trust; Leeds Teaching Hospitals NHS Trust; Mid Cheshire Hospitals NHS Foundation Trust; Moorfields Eye Centre at Bedford Hospital NHS Trust*; Moorfields Eye Centre at Croydon Health Services NHS Trust*; Nottingham University Hospitals NHS Trust; Norfolk and Norwich University Hospitals NHS Foundation Trust; North West Anglia NHS Foundation Trust; Northern Devon Healthcare NHS Trust; Oxford University Hospitals NHS Foundation Trust; Plymouth Hospitals NHS Trust; Portsmouth Hospitals NHS Trust; Royal Berkshire NHS Foundation Trust; Royal Cornwall Hospitals NHS Trust; Royal Free London NHS Foundation Trust; Royal United Hospitals Bath NHS Foundation Trust; Salisbury NHS Foundation Trust; Sheffield Teaching Hospitals NHS Foundation Trust; Sherwood Forest Hospitals NHS Foundation Trust; Shrewsbury and Telford Hospital NHS Trust; South Tees Hospitals NHS Foundation Trust; South Warwickshire NHS Foundation Trust; Southport and Ormskirk Hospital NHS Trust; St Helens and Knowsley Teaching Hospitals NHS Trust; Stockport NHS Foundation Trust; The Hillingdon Hospitals NHS Foundation Trust; East Suffolk and North Essex NHS Foundation Trust; The Newcastle upon Tyne Hospitals NHS Foundation Trust; The Mid Yorkshire Hospitals NHS Trust; The Princess Alexandra Hospital NHS Trust; The Royal Bournemouth and Christchurch Hospitals NHS Foundation Trust; Torbay and South Devon NHS Foundation Trust; University Hospital Southampton NHS Foundation Trust; University Hospitals Birmingham NHS Foundation Trust; University Hospitals Bristol NHS Foundation Trust; University Hospitals Coventry and Warwickshire NHS Trust; University Hospitals of Morecambe Bay NHS Foundation Trust; Warrington and Halton Hospitals NHS Foundation Trust; Wirral University Teaching Hospital NHS Foundation Trust; Wrightington, Wigan and Leigh NHS Foundation Trust; Wye Valley NHS Trust; Yeovil District Hospital NHS Foundation Trust; Plus the following SpaMedica centres providing NHS funded cataract surgery, Bolton; Liverpool; Manchester; Newton-le-Willows; Wakefield; Wirral; *These two centres data have been combined as they are part of the same governing authority for ophthalmology.

\section{Compliance with ethical standards}

Conflict of interest This study was supported through The Healthcare Quality Improvement Partnership (HQIP) commissioned Royal
College of Ophthalmologists (RCOphth) National Ophthalmology Database Audit, which is part of the National Clinical Audit and Patient Outcomes Programme (NCAPOP) and the Clinical Outcomes Programme (COP). All authors have completed the ICMJE uniform disclosure form at www.icmje.org/coi_disclosure.pdf and declare: PHJD, RJ (deceased) and JMS had financial support from HQIP for the submitted work; RJ was previously a director of Medisoft, the provider of the EMR with which the majority of the data for this study were collected; no other financial relationships with organisations that might have an interest in the submitted work in the previous three years; no other relationships or activities that could appear to have influenced the submitted work. ACD was supported by the National Institute for Health Research (NIHR) Biomedical Research Centre based at Moorfields Eye Hospital NHS Foundation Trust and UCL Institute of Ophthalmology. The views expressed are those of the author(s) and not necessarily of the NHS, the NIHR, HQIP or the Department of Health.

Publisher's note: Springer Nature remains neutral with regard to jurisdictional claims in published maps and institutional affiliations.

\section{References}

1. GOV.UK. The NHS Constitution for England - GOV.UK. Available at: https://www.gov.uk/government/publications/thenhs-constitution-for-england/the-nhs-constitution-for-england (Accessed 15 Sept 2017).

2. Keenan T, Rosen P, Yeates D, Goldacre M. Time trends and geographical variation in cataract surgery rates in England: study of surgical workload. Br J Ophthalmol 2007;91:901-4.

3. Coronini-Cronberg S, Lee H, Darzi A, Smith P. Evaluation of clinical threshold policies for cataract surgery among English commissioners. J Health Serv Res Policy 2012;17:241-7.

4. Whillans J, Nazroo J. Equal access, (Un)equal uptake: a longitudinal study of cataract surgery uptake in older people in England. BMC Health Serv Res 2014;14:447.

5. Coronini-Cronberg S, Bixby H, Laverty AA, Wachter RM, Millett C. English National Health Service's savings plan may have helped reduce the use of three 'low-value' procedures. Health Aff 2015;34:381-9.

6. GOV.UK. English indices of deprivation 2015 - GOV.UK. Available at: https://www.gov.uk/government/statistics/englishindices-of-deprivation-2015 (Accessed 16 Sept 2017).

7. Younan C, Mitchell P, Cumming R, Rochtchina E. Socioeconomic status and incident cataract surgery: the Blue Mountains Eye Study. Clin Exp Ophthalmol 2002;30:163-7.

8. Smirthwaite G, Lundström M, Wijma B, Lykke N, Swahnberg K. Inequity in waiting for cataract surgery-an analysis of data from the Swedish National Cataract Register. Int $\mathbf{J}$ Equity Health 2016;15:10.

9. Broman AT, Hafiz G, Muñoz B, Rodriguez J, Snyder R, Klein R, et al. Cataract and barriers to cataract surgery in a US Hispanic population: Proyecto VER. Arch Ophthalmol 2005;123:1231-6.

10. Wesolosky JD, Rudnisky CJ. Relationship between cataract severity and socioeconomic status. Can J Ophthalmol 2013;48:471-7. 